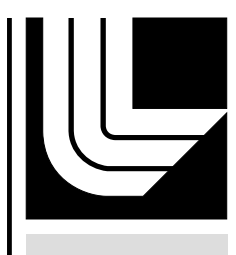

LAW RENCE LIVERMORE N A T IO N A L LABORATORY

\title{
UCRL-TR-235978
}

\section{Wavelet Denoising of Mobile Radiation Data}

D. Campbell, R. Lanier

October 30, 2007 
This document was prepared as an account of work sponsored by an agency of the United States government. Neither the United States government nor Lawrence Livermore National Security, LLC, nor any of their employees makes any warranty, expressed or implied, or assumes any legal liability or responsibility for the accuracy, completeness, or usefulness of any information, apparatus, product, or process disclosed, or represents that its use would not infringe privately owned rights. Reference herein to any specific commercial product, process, or service by trade name, trademark, manufacturer, or otherwise does not necessarily constitute or imply its endorsement, recommendation, or favoring by the United States government or Lawrence Livermore National Security, LLC. The views and opinions of authors expressed herein do not necessarily state or reflect those of the United States government or Lawrence Livermore National Security, LLC, and shall not be used for advertising or product endorsement purposes.

This work performed under the auspices of the U.S. Department of Energy by Lawrence Livermore National Laboratory under Contract DE-AC52-07NA27344. 


\title{
NNSA Office of Emergency Response FY08 Technology Integration Project Interim Report
}

\author{
Wavelet Denoising of Mobile Radiation Data \\ LLNL-2006-25-05 \\ D.B. Campbell, Ph.D. ${ }^{1}$ \\ R. Lanier, Ph.D.
}

\author{
${ }^{1}$ Contact Information \\ Lawrence Livermore National Laboratory \\ P.O. Box 808 \\ Mail Stop: L-171 \\ Livermore, CA 94551 \\ Phone: 925-422-5271 \\ FAX: 925-424-3101 \\ e-mail: campbell61@1lnl.gov
}




\begin{abstract}
The investigation of wavelet analysis techniques as a means of filtering the grosscount signal obtained from radiation detectors has shown promise. These signals are contaminated with high frequency statistical noise and significantly varying background radiation levels. Wavelet transforms allow a signal to be split into its constituent frequency components without losing relative timing information. Initial simulations and an injection study have been performed. Additionally, acquisition and analysis software has been written which allowed the technique to be evaluated in real-time under more realistic operating conditions. The technique performed well when compared to more traditional triggering techniques with its performance primarily limited by false alarms due to prominent features in the signal. An initial investigation into the potential rejection and classification of these false alarms has also shown promise.
\end{abstract}

\title{
Introduction
}

\section{Challenges of Mobile Radiation Detection}

The task of detecting radioactive sources for homeland security applications is a complex one. With limited assets and a massive area to protect, access to effective mobile radiation detection systems is extremely desirable. These systems can be deployed as needed based on individual threats rather than statically positioned to address the most likely scenarios. Additionally, mobile systems can search large areas rather than simply monitoring their immediate vicinity. In spite of the need for this capability, effective mobile systems have proven difficult to develop. The chief obstacle remains the dynamic nature of background radiation encountered by moving detectors. It is painfully clear that ambient radiation levels vary significantly from location to location. While stationary detectors can adjust to the levels associated with their current surroundings, mobile detectors are forced to continuously reassess background in a changing environment.

Fluctuations in background can vary dramatically. Deviations can range from gradual, perhaps due to the slow rise of terrain around the vehicle, to sudden such as an adjustment in count rate resulting from a change in the direction of motion. In the middle are a whole range of features in the signal from things such as changes in construction material and the existence of obstructing objects, such as large trucks. These deviations vary not only in magnitude and duration but also in regularity of occurrence. As a result, the small peaks associated with legitimate targets can quickly be swamped in background fluctuations.

The problems associated with the dynamic nature of the background encountered by moving radiation detectors has yet to be satisfactorily solved. The challenge is of sufficient complexity that every dimension of the signal should be evaluated. While spectral analysis is a powerful technique, there is also a significant amount of information in the temporal signal. Wavelets are a powerful technique to exploit this temporal information and complement analysis performed in the spectral dimension.

\section{Wavelet Fundamentals}

Wavelet analysis is similar to Fourier analysis, where a signal is broken down into sinusoids of different frequency. However, wavelet analysis allows frequency components to be localized in time. Additionally, wavelets are more adept than Fourier analysis at dealing with the challenges of mobile radiation data, such as discontinuities. 
Simply put, a wavelet is a waveform of limited duration with zero net area; the simplest example is shown in Figure 1. The first step in wavelet analysis is the selection of an appropriate "mother wavelet." Next the mother wavelet is successively compared to each section of the signal to be analyzed. For each point in the signal, a correlation coefficient is generated whose magnitude reflects how well the waveform mirrors the shape of the signal at that point. For the next iteration the wavelet is essentially stretched out, or dilated, using its corresponding scaling function. This process shifts the wavelet's sensitivity toward lower frequencies, or broader features. Then, the correlation procedure is repeated to localize the corresponding broad features in the signal.
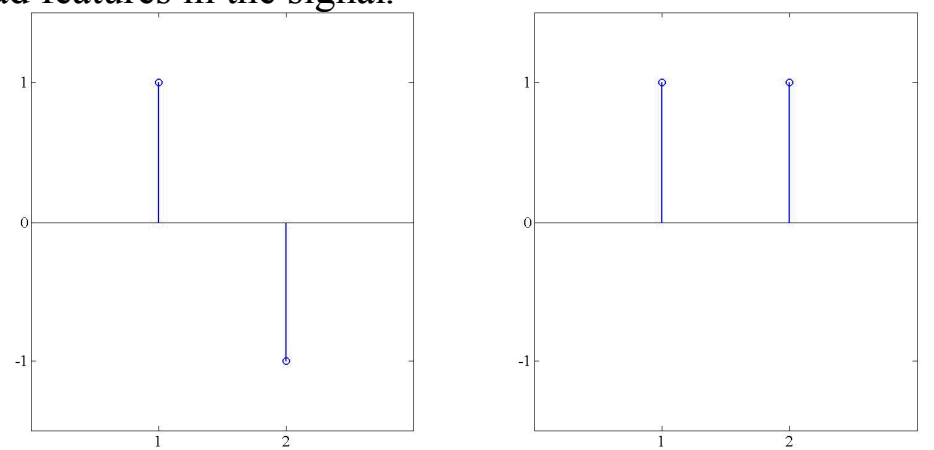

Figure 1: Left: The Haar wavelet. Right: The scaling function for the Haar wavelet.

The Db4 wavelet, from the Daubechies family of wavelets, has been selected for the current work. This collection of wavelets is widely used due to their robustness, large number of vanishing moments, and sensitivity to signals with both time and frequency characteristics. The Db4 wavelet contains four points $(-0.129,-0.224,0.837$, and -0.483$)$ and has two vanishing moments, see Figure 2. These vanishing moments mean the Db4 wavelet correlates poorly with both flat and sloped signals but is sensitive to trends shaped like higher order polynomials.
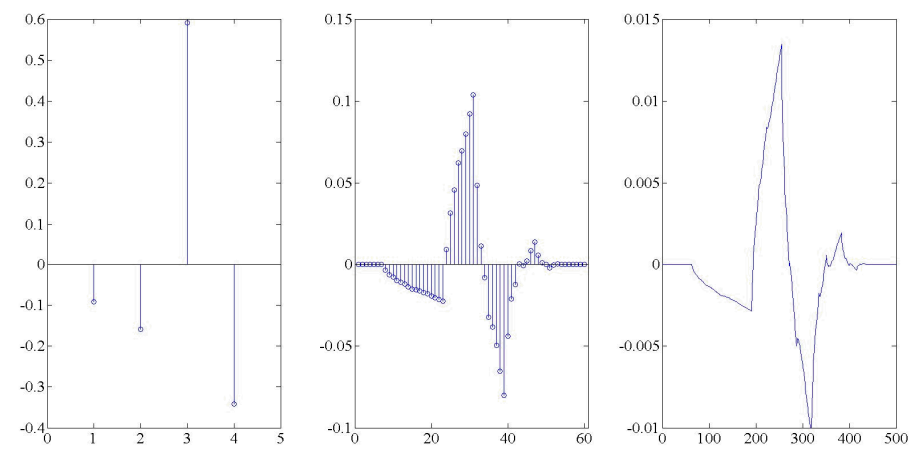

Figure 2: The $\mathrm{Db} 4$ wavelet at different dilations with 4, 60, and 508 points.

The continuous wavelet transform (CWT) has also been selected for the current work. With this technique the wavelet is scaled by adding one additional point per iteration. This is done by first constructing a gross waveform from the wavelet by scaling to the point where the wavelet is virtually continuous over its length. Then the waveform is sampled at the appropriate number of points to generate the wavelet of a desired size. For example, a Db4 wavelet with the required number of points could be constructed through simply sampling the waveform shown in the right panel of Figure 2. The CWT produces a matrix of correlation coefficients which indicate the presence of features of the corresponding size at 
each location in the signal. One possible triggering algorithm is simply to look for features of the appropriate frequency by monitoring a particular dilation, or width, of the wavelet. Figure 3 shows an example of isolating a peak of interest from a complicated background which would prove challenging to triggering methods based on rolling estimations of background.
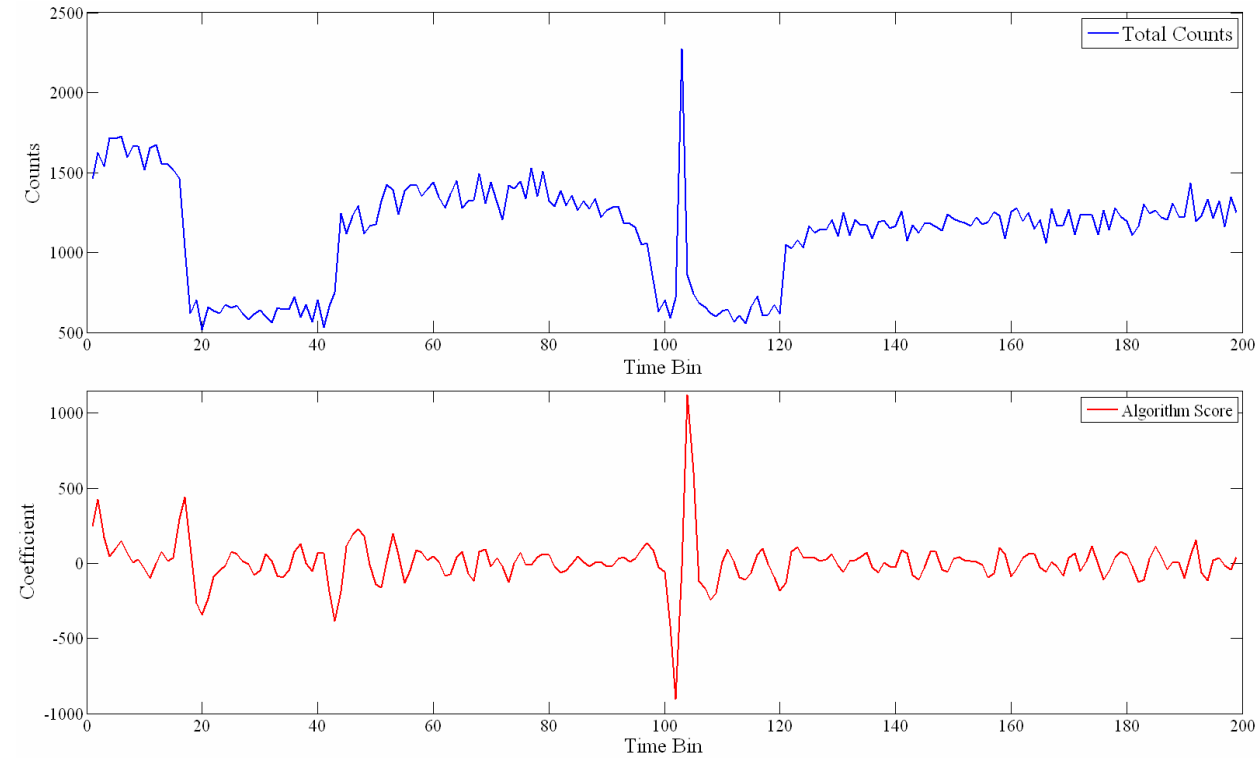

Figure 3: Top: Raw total counts as a function of time. Bottom: Isolation of a peak of the desired frequency.

\section{Injection Study}

Project

In order to gauge the performance of a triggering algorithm based on wavelet analysis an injection study was performed. This study utilized a set of mobile radiation data taken with a 2" by 4" by 16 " $\mathrm{NaI}(\mathrm{Tl})$ crystal mounted in a van and operated at a sample rate of 7 Hz. Each section of the data was evaluated once as it was collected and then again after the profile of a passing source as injected. Alarms which would have been triggered in the raw data constituted false alarms. Similarly, alarms reported by the algorithm on the data containing an injected source where used to calculate the probability of detection. A basic wavelet analysis algorithm consisting of monitoring the maximum correlation coefficient reported for each time bin was analyzed. For comparison, a standard sigma above background approach was also evaluated, with background estimated by a rolling 2 minute average. Receiver operating characteristic (ROC) curves were generated to aid in comparing the two algorithms.

\section{$\underline{\text { Results }}$}

The two ROC curves generated from the injection study are shown in Figure 4. The elementary wavelet algorithm (red) shows a clear edge in performance over the more standard approach (blue). This performance is not currently limited by the weakness of the injected signal but rather by background features which produce false alarms. While wavelets are a powerful technique for finding weak features in the data, they also identify features which should not trigger alarms. 


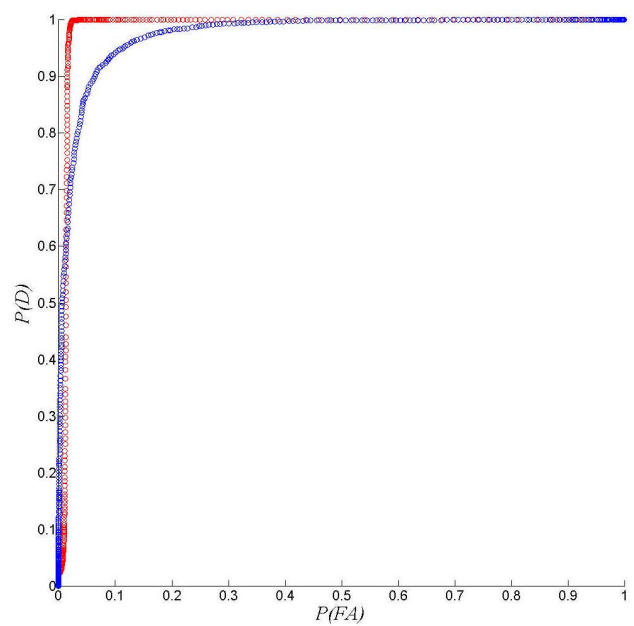

Figure 4: Receiver operating characteristic curves for the wavelet algorithm (red) and a standard n-sigma algorithm (blue).

A sample segment of mobile data is shown in Figure 5. The top panel shows the total counts as a function of time while the bottom panel shows the results produced by wavelet analysis. The basic triggering algorithm described above would trigger on the magnitudes of the peaks isolated from the background, with a possible triggering threshold shown with the red dashed line. Numerous small features not readily observed in the raw signal are evident in the wavelet output. However, some broader and stronger features are present as well and would trigger alarms if the threshold was set for maximal sensitivity.

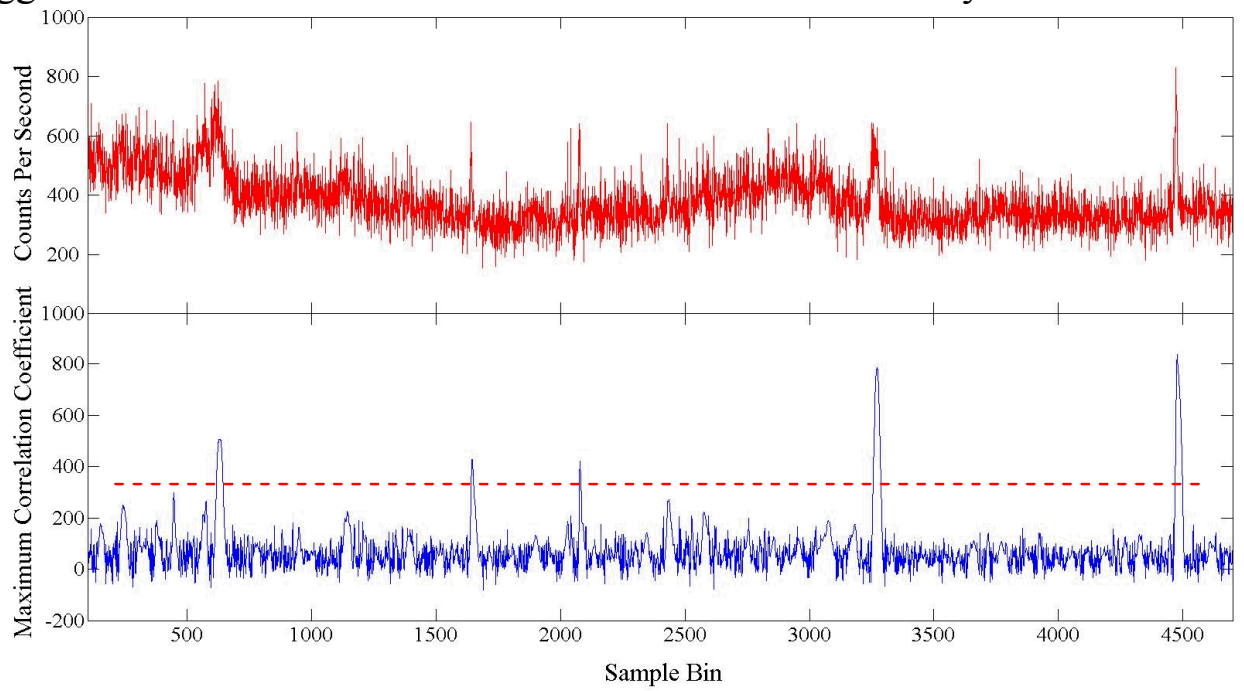

Figure 5: Top: A sample segment of mobile radiation data. Bottom: The signal after wavelet analysis with a possible trigger threshold shown by the dashed red line.

One possible solution to this limitation is to utilize more of the information produced by the wavelet analysis to design a more robust algorithm. For example, each of the features present in the bottom panel of Figure 5 can be classified based on a range of parameters obtained from the matrix of correlation coefficients produced by the continuous wavelet transform. The initial evaluation of this technique is detailed below.

The injection study also emphasized the importance of sample rate when performing wavelet analysis of mobile radiation data. Slower rates obscured signal details and diminish the usefulness of wavelets. A sample rate of $1 \mathrm{~Hz}$ is fairly common for commercial radiation 
detection systems. As a result, a large data set of previously obtained mobile radiation data proved less useful than initially anticipated due to loss of detail. As an example, the distinguishability of peaks generated by sources at different speeds is plotted for both $10 \mathrm{~Hz}$ and $1 \mathrm{~Hz}$ sample rates in Figure 6. While broad classifications can be made with the faster sampling rate, the wavelet analysis performs much worse with the slow sampling rate.

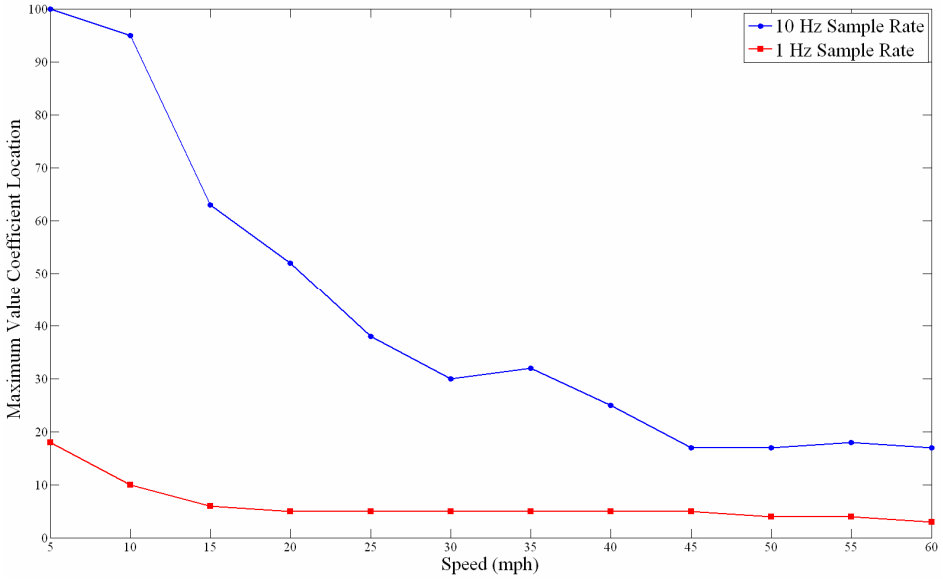

Figure 6: The effect of sampling rate on the ability of wavelets to distinguish source profiles based on speed.

This poor performance resulted from the similarity of profiles generated by sources moving at or above moderate speeds. When sampled too slowly, profiles essentially became peaks 1 time-bin wide. As wavelets are sensitive to the bin width of features, the result of the analysis was roughly identical when the data was binned too coarsely. An example is plotted in Figure 7 where a profile generated by a $1 \mathrm{mCi}$ source traveling at $20 \mathrm{mph}$ and an impact parameter of 24 feet is sampled at $10 \mathrm{~Hz}$ and $1 \mathrm{~Hz}$. The loss of structure is evident as the width of the peak is condensed from rough twenty to two.
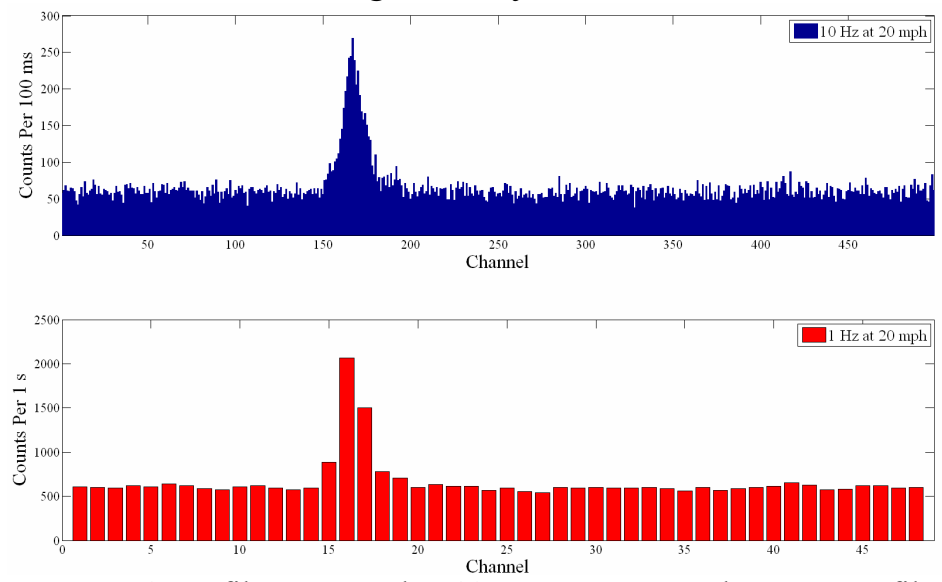

Figure 7: Top: A profile generated at $10 \mathrm{~Hz}$. Bottom: The same profile at $1 \mathrm{~Hz}$.

\section{Real Time Analysis}

Project

Analysis and acquisition software was written to implement the algorithm in real-time in an effort to explore the challenges posed by more realistic operating conditions. The MatLab development environment was chosen to facilitate analysis. The analysis software was capable of operating a $\mathrm{NaI}(\mathrm{Tl})$ crystal through a universal serial bus (USB) connection to an 
ORTEC digiBASE multi-channel analyzer. Compatibility with a GPS antenna was also incorporated to track vehicle speed and location.

\section{$\underline{\text { Results }}$}

The chosen method for data acquisition and analysis proved highly functional during data collection and algorithm development. New concepts could be implemented rapidly with a minimum of coding. Some minimal latency issues were encountered due to computational overhead and less than optimum performance of the development environment. A sample screen shot of the graphical user interface (GUI) is shown in Figure 8.

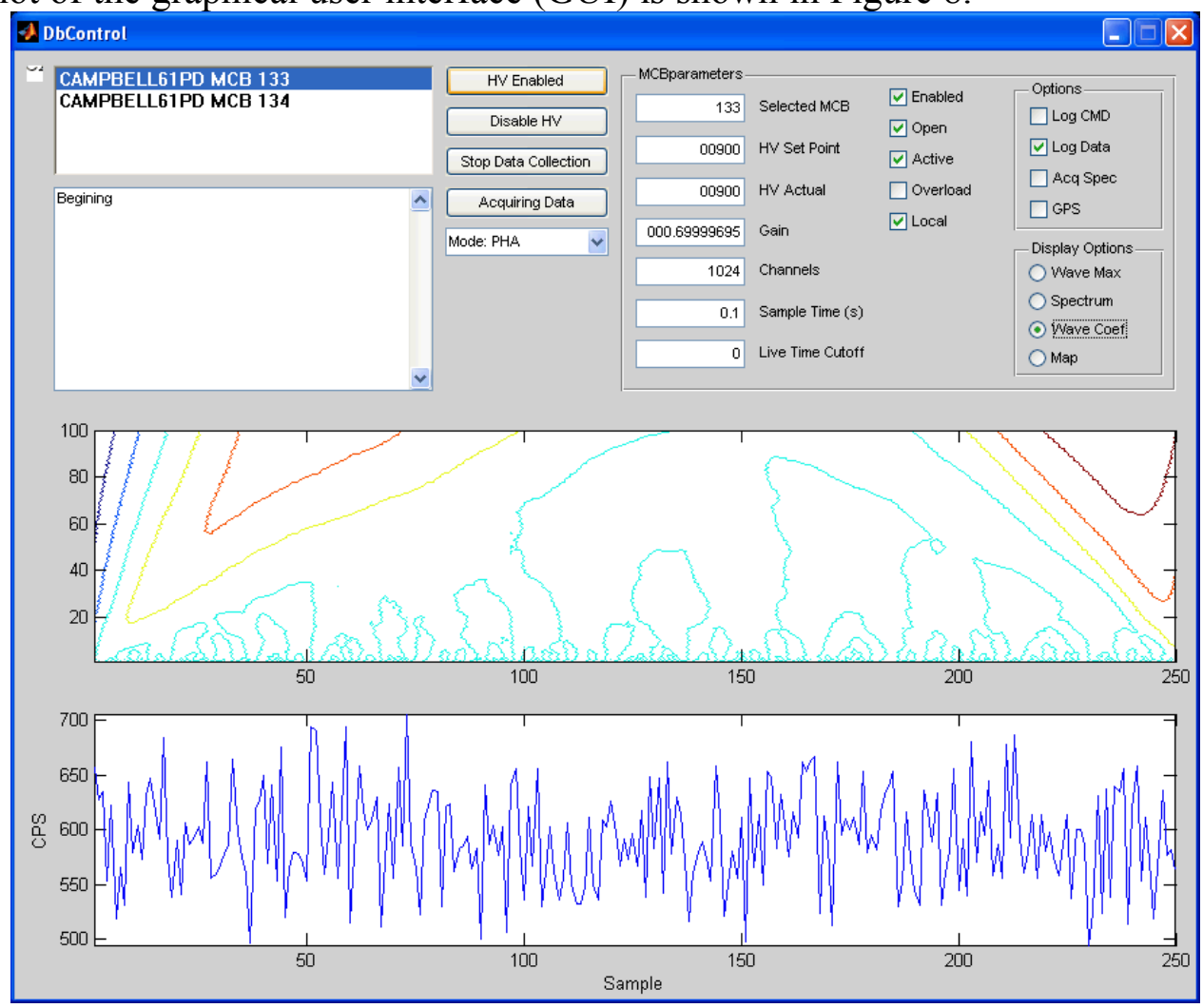

Figure 8: A screen capture of the analysis software GUI.

Two plots are shown in the GUI in Figure 8. The bottom plot is a trace of the total counts signal of the $\mathrm{NaI}(\mathrm{Tl})$ detector. The top plot is a contour of the matrix of correlation coefficients produced for the total counts signal shown. The first 100 dilations, or widths, of the Db4 wavelet are shown with wide (low frequency) instances of the wavelet at the top and narrow (high frequency) instances at the bottom. The most prominent characteristic shown in the coefficient matrix are the large values present at the beginning and end of the signal. These values are produced as the increasingly wider wavelets higher up the y-axis span the edge of the signal. These persistent edge effects are roughly constant and can be corrected.

Figure 9 shows two screen captures of the analysis GUI, one for a high frequency peak and one for a lower frequency structure. The coefficient matrix clearly shows a shift in the width of the wavelet which most closely matches the signal. The edge effects have been subtracted in both panels. These patterns shown in the coefficient matrix contain a great deal of information about the corresponding signal and could potentially be used to reduce the occurrence of false alarms. 


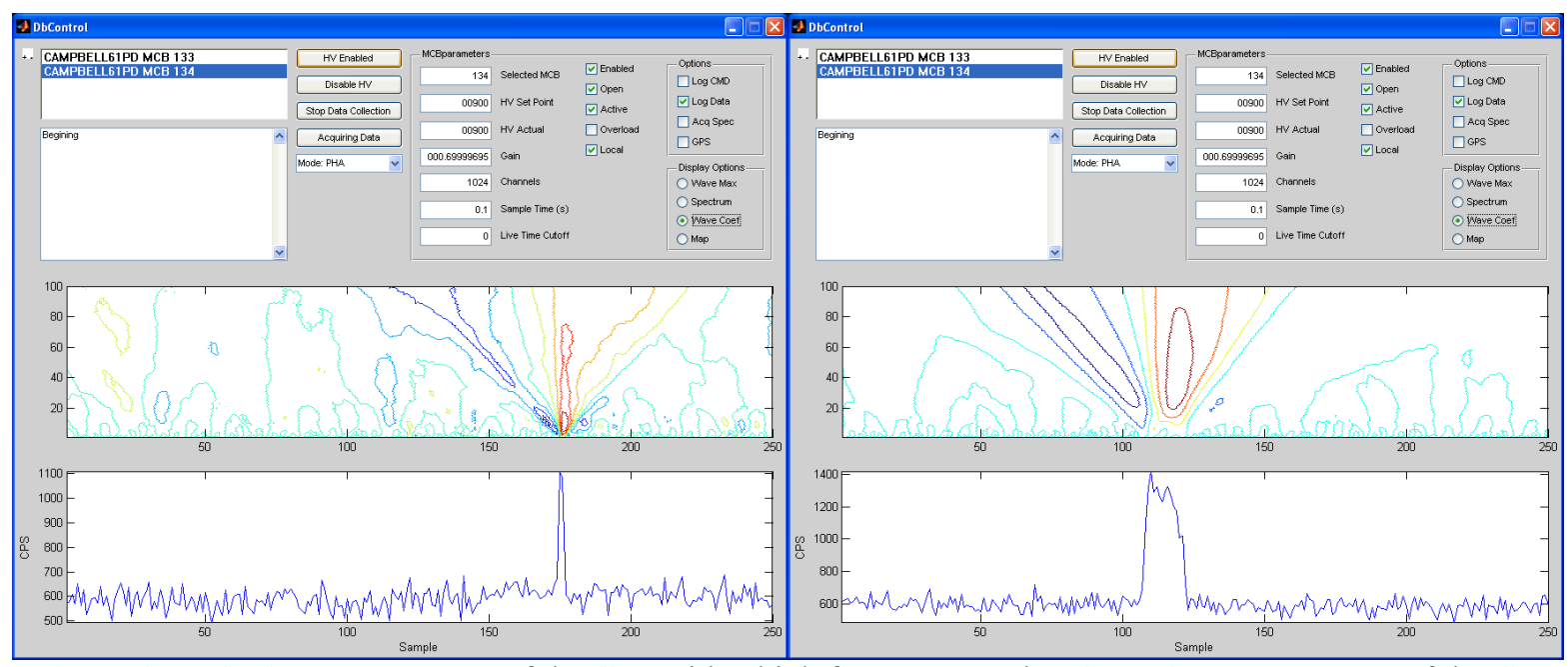

Figure 9: Left: A screen-capture of the GUI with a high frequency peak. Right: A screen-capture of the GUI with a lower frequency peak.

\section{Initial Evaluation of Pattern Recognition for Algorithm Enhancement}

\section{$\underline{\text { Project }}$}

While scanning the total-counts signal for features which match the expected profile of a passing source has merit, the injection study highlighted the more challenging situation of coping with complicated profiles. The situation shown in Figure 10 is an example of a profile which does not conform to a simple source trajectory.
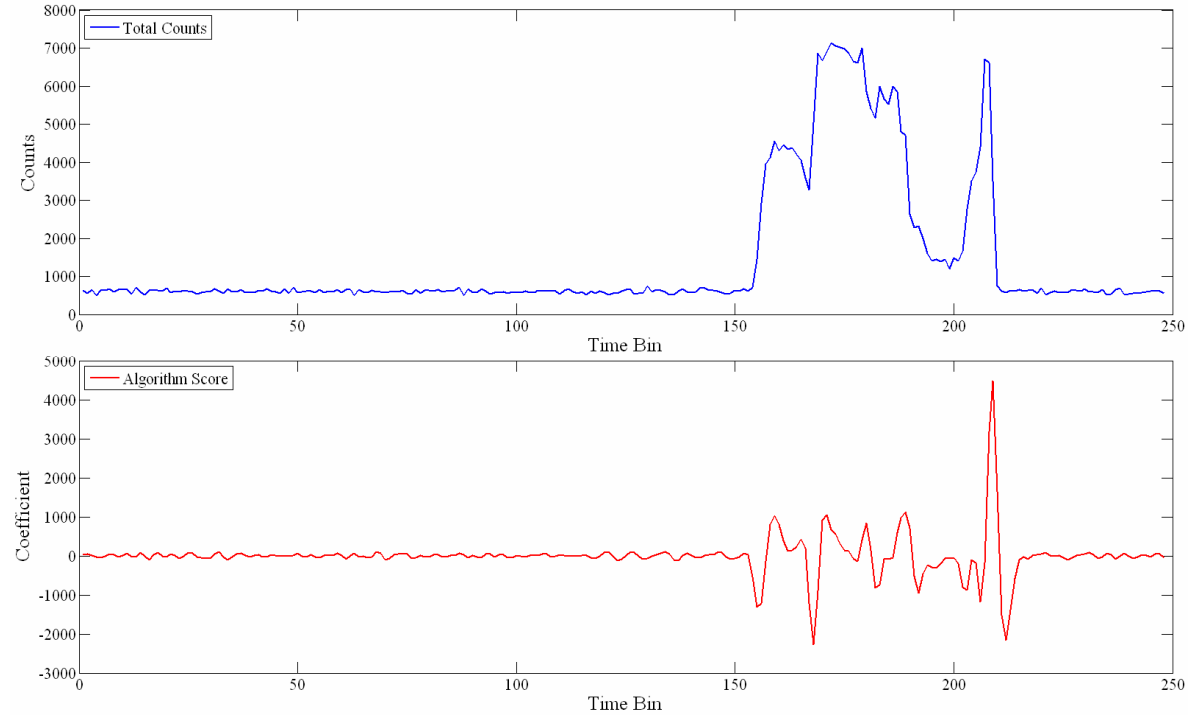

Figure 10: Top: A complicated total counts signal. Bottom: The algorithm score produced by monitoring a coefficient tuned to find narrow peaks.

The initial wavelet algorithm does well to isolate the desired feature while not triggering off of the leading edge of the profile. However, as shown in Figure 9, the coefficient matrix produced by the CWT offers significantly more information about the shape of the total counts signal. A preliminary examination of profile classification based on the coefficient matrix was performed. The separability of point sources as well as the feasibility of extracting features from complicated profiles to perform classification was evaluated. 


\section{$\underline{\text { Results }}$}

Several key parameters which were easily obtained from the coefficient matrix where evaluated for each signal. These features included:

- the location of the wavelet dilation with the highest correlation coefficient

- the location of the wavelet dilation with the lowest correlation coefficient

- the Cartesian separation of these minima and maxima

- the area around the maximum as defined by a $90 \%$ of max threshold

A set of point source profiles and a set of exotic non-point source profiles were evaluated. The set of point source profiles spanned from very narrow peaks to peaks which closely resembled square waves. A sample of results is plotted in Figure 11.
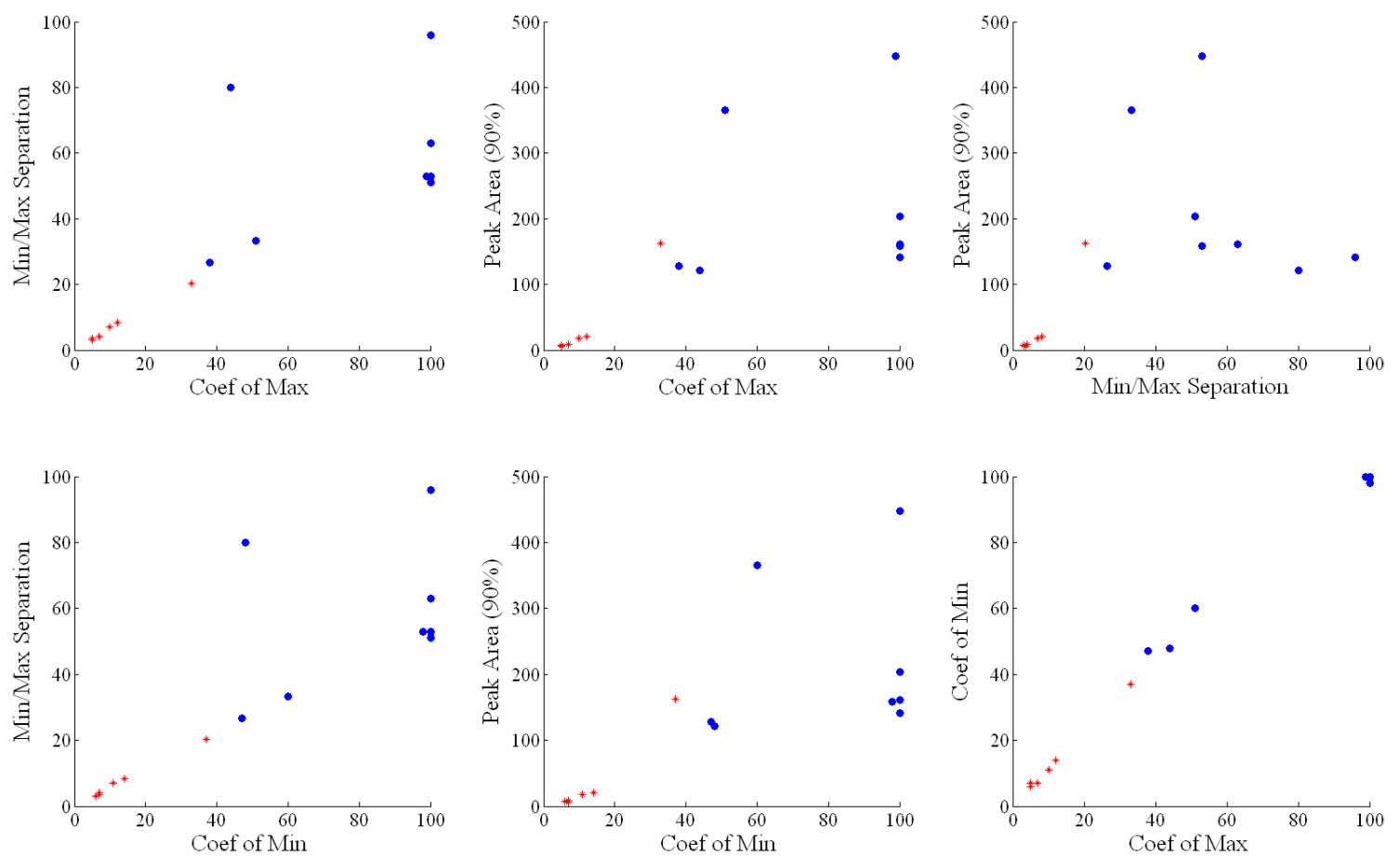

Figure 11: Comparisons between select parameters for point source profiles (red) and non-point source profiles (blue).

The point sources are well grouped and clearly separable from the non-point sources. These results are promising given the rudimentary nature of the analysis and suggest there is merit in attempting a more refined analysis of the coefficient matrix. More powerful pattern recognition techniques may allow these complicated source profiles to be grouped into broad categories. This clustering could aid in rejecting false alarms as well as offer additional insight to aid system operators.

\section{Programmatics}

FY07 Milestones

- Injection Study

- Interim Report Delivered at mid-year review

- Real Time Analysis

- Final Report 


\section{Funding Status}

- FY07: \$1k remaining

- FY08: \$140k to investigate pattern recognition techniques on the wavelet analysis output to reject false alarms as well as correlate radiation and video data

\section{Conclusion}

Wavelets have proven to be a powerful tool for analyzing data from mobile radiation detectors. Analysis was evaluated in real-time and in post-processing. Initial algorithms performed well when compared to more traditional techniques. These initial algorithms excelled at extracting weak features from the total-counts signal of a moving detector. However, false alarm rates were elevated as additional features were also extracted.

Wavelets are a quick and effective method for quantifying the characteristics of complex signal profiles. The coefficient matrix produced by the CWT has proven valuable for analysis based on feature extraction and shows potential as a basis for more sophisticated pattern recognition techniques. This capability should allow the exploitation of orthogonal data, such as video cameras, and the automation of expert analysis techniques. These automated routines could be used to potentially reduce false alarm rates as well as effectively identify targets for secondary screening. 